\title{
The environment of barred galaxies revisited
}

\author{
Bernardo Cervantes Sodi, Cheng Li, Changbom Park \& Lixin Wang
}

\begin{abstract}
We present a study of the environment of barred galaxies using galaxies drawn from the SDSS. We use several different statistics to quantify the environment: the projected two-point cross-correlation function, the background-subtracted number count of neighbor galaxies, the overdensity of the local environment, the membership of our galaxies to galaxy groups to segregate central and satellite systems, and for central galaxies we estimate the stellar to halo mass ratio $\left(\mathrm{M}_{*} / \mathrm{M}_{\mathrm{h}}\right)$ . When we split our sample into early- and late-type galaxies, we see a weak but significant trend for early-type galaxies with a bar to be more strongly clustered on scales from a few $100 \mathrm{kpc}$ to $1 \mathrm{Mpc}$ when compared to unbarred early-type galaxies. This indicates that the presence of a bar in early-type galaxies depends on the location within their host dark matter halos. This is confirmed by the group catalog in the sense that for early-types, the fraction of central galaxies is smaller if they have a bar. For late-type galaxies, we find fewer neighbors within $\sim 50 \mathrm{kpc}$ around the barred galaxies when compared to unbarred galaxies from the control sample, suggesting that tidal forces from close companions suppress the formation/growth of bars. For central late-type galaxies, bars are more common on galaxies with high $\mathrm{M}_{*} / \mathrm{M}_{\mathrm{h}}$ values, as expected from early theoretical works which showed that systems with massive dark matter halos are more stable against bar instabilities. Finally, we find no obvious correlation between overdensity and the bars in our sample, showing that galactic bars are not obviously linked to the large-scale structure of the universe.
\end{abstract}

Bernardo Cervantes Sodi

Korea Institute for Advance Study, Dongdaemun-gu, Seoul 130-722, Republic of Korea, and Centro de Radioastronomía y Astrofísica - UNAM, Apdo. Postal 3-72 (Xangari), 58089 Morelia, Mich., Mexico, e-mail: b.cervantes@crya.unam.mx

Cheng Li \& Lixin Wang

Key Laboratory for Research in Galaxies and Cosmology of Chinese Academy of Sciences, Shanghai Astronomical Observatory, Nandan Road 80, Shanghai 200030, China

Changbom Park

Korea Institute for Advanced Study, Dongdaemun-gu, Seoul 130-722, Korea 


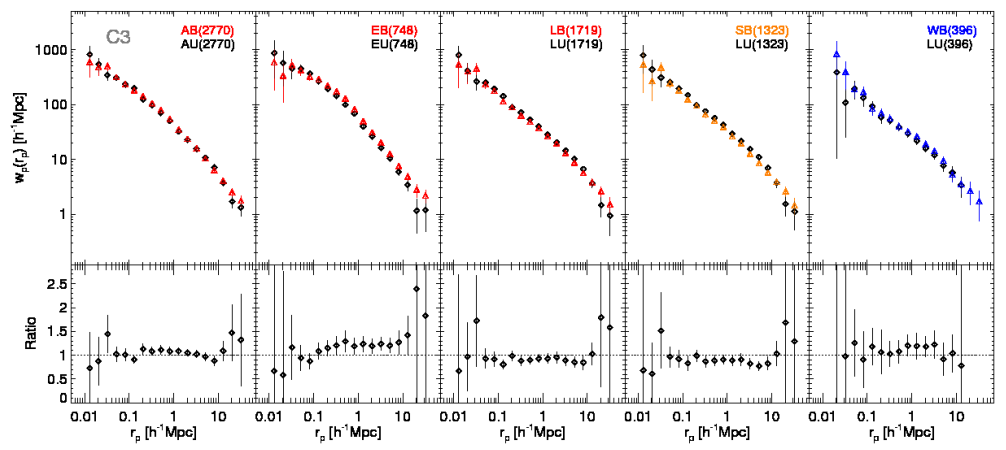

Fig. 1 Projected 2PCCF for barred $w_{p}^{\text {bar }}\left(r_{p}\right)$ and unbarred $w_{p}^{\text {unbar }}\left(r_{p}\right)$ galaxies (top panels) and $w_{p}^{\text {bar }}\left(r_{p}\right)$ to $w_{p}^{\text {unbar }}\left(r_{p}\right)$ ratio (bottom panels) for the different subsamples of our control sample C3 where the galaxies share a common distribution of stellar mass $M_{*}$, color $\mathrm{g}-\mathrm{r}$, and stellar surface mass density $\mu_{*}$. Panels from left to right correspond to the whole galaxy sample of barred ( $\mathrm{AB}$ orange triangles) plus unbarred (AU black diamonds) galaxies, early-types barred (EB orange triangles) and unbarred (EU black diamonds), late-types barred (LB orange triangles) and unbarred (LU black diamonds), strongly barred late-types (SB yellow triangles) and LU, and weakly barred late-types (WB blue triangles) and LU.

\section{Background}

With the outcome of large galaxy surveys, the role of environment in triggering or suppressing the formation of stellar bars in galaxies has been a popular topic of research, with a large variety of results, depending on the methods employed [7, 4, 3, 6].

In this work we employ a large volume-limited sample of $\sim 30,000$ galaxies to study the likelihood for galaxies to host bars as a function of environment, using several statistics to study different scales, with the aim of giving a complete study for galaxies in the nearby universe. For a more detailed description of the sample and a complete discussion of the results, we refer the reader to [5, 1].

\section{Main Results}

Our first approach is to compute the two-point cross correlation function of our galaxy sample with respect to a reference sample of the general galaxy population (2PCCF, $w_{p}\left(r_{p}\right)$ ), from scales of a few tens of kiloparsecs up to a few tens of megaparsecs. We then construct a control sample of unbarred galaxies with matched stellar mass, color and surface mass density $\left(\triangle \log M_{*} \leq 0.08, \triangle(g-r) \leq 0.025\right.$, $\left.\triangle \log \mu_{*} \leq 0.08\right)$. In Figure 1, first panel, we present the $2 \mathrm{PCCF}$ for the barred sample (AB), the control sample (AU) as well as the ratio between them. In this 


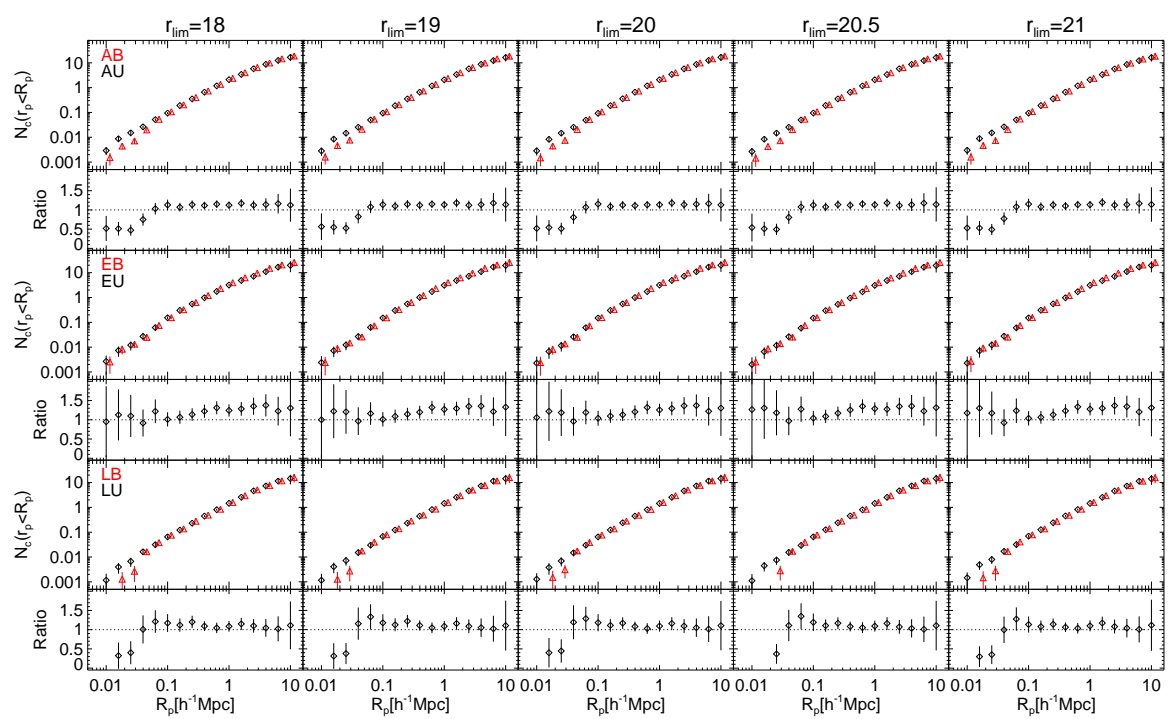

Fig. 2 Average counts of galaxies in the photometric sample within a given projected radius $\mathrm{R}_{P}$ from the galaxies in our samples of barred and unbarred control samples. Each line corresponds to different apparent magnitude limits in r-band $(r \leq 18,19,20,20.5,21)$ for the galaxies in the photometric sample.

case, no significant overclustering is detected for the full sample of barred galaxies over unbarred ones, but if we segregate the samples by morphology, the 2PCCF for early-type barred galaxies (EB) show a weak but significant trend of overclustering on scales from a few $100 \mathrm{kpc}$ to $1 \mathrm{Mpc}$, when compared with unbarred early-type galaxies (EU), a trend not found with the late-type galaxies, regardless if their bars are classified as strong or weak. This result implies that the presence of bars in earlytype galaxies depends on the location of the galaxies within their host dark matter halos.

With the fiber collision problem suffered by the SDSS at small separation angles, we explore the clustering of our samples at small separations by computing the background-subtracted neighbor counts, $N_{C}\left(r_{p}<R_{p}\right)$, which is the number of galaxies in the photometric reference sample within the projected radius $R_{p}$ of the barred or unbarred galaxies, with the effect of chance projection being statistically corrected [5]. In Figure 2 we plot $N_{C}$, panels top to bottom show the results for the whole sample, the early-type and late-type subsamples respectively and from left to right, the results for different apparent $r$-band limiting magnitude applied to the photometric reference sample, ranging from $r_{\text {lim }}=18$ for the left-most panels to $r_{l i m}=21$ for the right-most panels. For early-type galaxies, the overclustering of barred galaxies becomes again evident for scales larges than $100 \mathrm{kpc}$, but this time the subsample including only late-type galaxies shows a lack of neighbors in the vicinity of barred galaxies on scales $\lesssim 50 \mathrm{kpc}$ when compared with unbarred 
galaxies, suggesting that tidal forces from close companions suppress the formation/growth of bars.
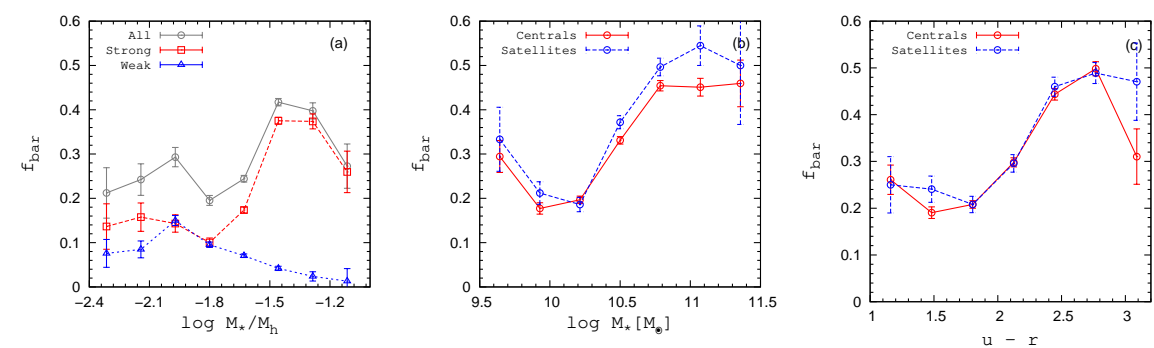

Fig. 3 The fraction of barred galaxies $f_{\text {bar }}$ as a function of (a) stellar-to-halo mass ratio, for strong, weak and strong plus weak bars, (b) stellar mass for central and satellite galaxies and, (c) color for centrals and satellites.

Using the galaxy catalog by [8], we classified our galaxies as central or satellite galaxies. For the late-type centrals, we estimate the stellar-to-halo mass ratio $\left(\mathrm{M}_{*} / \mathrm{M}_{\mathrm{h}}\right)$, using the stellar mass data from the MPA/JHU SDSS database [2] and the halo masses from the same galaxy catalog. Figure 3 (a) shows that the bar fraction is a strong function of this ratio, with strong bars more commonly found in galaxies with high $\mathrm{M}_{*} / \mathrm{M}_{\mathrm{h}}$, as expected form theoretical works which show that galaxies with massive dark matter halos are more stable against bar formation, while weak bars follow the opposite trend.

With our galaxies segregated into centrals and satellites, we compare the bar fraction of both subsamples as a function of stellar mass and color in Figure 3(b,c). At fixed stellar mass we find that the bar fraction of satellite galaxies is slightly higher than that of central galaxies, but at fixed color this difference vanishes. We interpret this as follows; the color of satellite galaxies, on average, is redder than the color of centrals at the stellar mass ranges involved in our study. Given that the bar fraction is higher for redder galaxies, the difference of the bar fraction between centrals and satellites is not directly due to the group environment, but indirectly through its dependence on internal morphology.

\section{References}

1. Cervantes Sodi, B., Li, C., \& Park, C. 2015, ApJ, 807, 111

2. Kauffmann, G., Heckman, T. M., White, S. D. M., et al. 2003, MNRAS, 341, 33

3. Lee, G.-H., Park, C., Lee, M. G., \& Choi, Y.-Y.: 2012, ApJ 745, 125

4. Li, C., Gadotti, D. A., Mao, S., \& Kauffmann, G.: 2009, MNRAS 397, 726

5. Lin, Y., Cervantes Sodi, B., Li, C., Wang, L., \& Wang, E.: 2014, ApJ, 796, 98

6. Skibba, R. A., Masters, K. L., Nichol, R. C., et al.: 2012, MNRAS, 423, 1485

7. van den Bergh, S.: 2002, ApJ, 124, 782

8. Yang, X., Mo, H. J., van den Bosch, F. C., et al.: 2007, ApJ, 671, 153 